\title{
On Three-Fold Coordinated Si Sites in Mesoporous MCM-41 Catalysts
}

\author{
J. Aguilar-Pliego ${ }^{*}, 1$ P. Bosch ${ }^{2}$, C. Zicovich-Wilson ${ }^{3}$, G. Herrera-Pérez ${ }^{4}$ and V.H. Lara ${ }^{5}$ \\ ${ }^{1}$ Universidad Autónoma Metropolitana-Azcapotzalco, San Pablo 180, 02200, D.F., México \\ ${ }^{2}$ Universidad Nacional Autónoma de México, Instituto de Investigaciones en Materiales, D.F,. México \\ ${ }^{3}$ Universidad Autónoma del Estado de Morelos, Morelos, México \\ ${ }^{4}$ Instituto Tecnológico de Estudios Superiores de Irapuato, Carretera Irapuato - Silao Km. 12.5, C.P. 36821 Irapuato, \\ Guanajuato, México \\ ${ }^{5}$ Universidad Autónoma Metropolitana-Iztapalapa, Depto. Química, D.F., México
}

\begin{abstract}
Si}-\mathrm{NMR}$, gas adsorption and SAXS results are discussed to determine if the geometry and the electronic properties of Si sites, present in the MCM-41walls, are more similar to three- than to four-fold coordinated sites. The features of Si-MCM-41, SBA-15, Y zeolite and silica $\left(\mathrm{SiO}_{2}\right)$, characterized by several techniques, are compared. It is shown that the pre-polymer geometries are similar to those of the zeolite secondary building units, but, in the organization-polymerization step, the generation of partially saturated or quite stressed structures is favored.
\end{abstract}

Keywords: MCM-41, porous materials, fractal, MCM-41 catalysts.

\section{INTRODUCTION}

The synthesis, in the 90 's, of a new family of mesoporous silica molecular sieves, the so-called M41S, has opened an interesting and promising field in material chemistry. These compounds, crystallized in presence of surfactants and basic medium [1], present high surface areas $\left(\sim 1000 \mathrm{~m}^{2} / \mathrm{g}\right)[2,3]$, and pore diameters that range from 20 to $50 \AA$. These pores are significantly larger than those present in other low-density silicates. Such remarkable features have been very appealing for technological applications such as catalysis $[4,5]$, separation processes or host-guest chemistry. They may be very useful for the synthesis of compounds with biological activity and large molecular size that could not be efficiently managed in other smaller pore materials, like zeolites, owing to steric hindrances with the cavity walls.

One of the most widely employed phases of the M41S family is the hexagonal MCM-41. Several studies have been devoted to the determination of the structural features of this material and its analogues [1-3]. It is now well established that, while their channel system is regular at the nanometric scale, the microscopic structure exhibits no appreciable order. Nonetheless, the short-range degree of disorder is still a matter of discussion. Indeed, it is difficult to obtain, from the usual characterization techniques, detailed information at the atomic scale, and to devise models suitable to perform "realistic" computational simulations of the formation processes.

Despite this lack of explicit data, it is currently assumed that corner-shared $\mathrm{SiO}_{4}$ units constitute the channel walls,

*Address correspondence to this author at the Universidad Autónoma Metropolitana-Azcapotzalco, San Pablo 180, 02200, D.F., México; Tel: (+55) 53182088; Fax: (+55)53189538; E-mail: apj@correo.azc.uam.mx just as they appear in siliceous zeolites and other ordered silicon oxides. However, to avoid structural collapse, in mesoporous silica, the occurrence of large void inner volumes requires walls much more rigid than those present in zeolites. Thus at the very short range, involving the primary building units, the structures must be significantly different.

In the present study, we discuss X-ray diffraction (XRD), Small-Angle X-ray Scattering (SAXS), ${ }^{29} \mathrm{Si}-\mathrm{NMR}$, gas (nitrogen and argon) adsorption results, as they may provide some clues on the occurrence of $\mathrm{Si}$ sites in the walls of MCM-41 whose geometry and electronic properties resemble more to three- than to four-fold coordinated sites. For the sake of comparison the SAXS and XRD experiments have been performed on samples of silica gel, SBA-15, MCM-41, and Al-MCM-41 porous materials as well as Y Faujasite zeolite.

\section{EXPERIMENTAL}

\section{Materials}

The Y zeolite was a commercial Y Faujasite (PQ zeolites $\mathrm{BV}$ ), with a Si/Al ratio of 15 . The synthesis of the MCM-41 was carried out by co-precipitation [4]. A $0.7 \% \mathrm{w} / \mathrm{w}$ aqueous solution of cetyltrimethylammonium (CTAB, Aldrich) was prepared and kept under stirring $(700 \mathrm{rpm})$ for the rest of the procedure. $500 \mathrm{ml}$ of $\mathrm{NH}_{4} \mathrm{OH}(30 \% \mathrm{w} / \mathrm{w})$ was added and stirred until a homogeneous mixture was formed, then aluminum salt $\left(\mathrm{Al}\left(\mathrm{NO}_{3}\right)_{3}\right.$, Aldrich, in the case of the samples with aluminum) was added to attain a $\mathrm{Si} / \mathrm{Al}$ ratio of 25. After 30 minutes, tetraethylsilicate (TEOS, Aldrich) was added, drop wise, and kept under stirring for 24 hours at room temperature. The resultant solid was filtered, washed, and dried for 12 hours at room temperature. The solid was, then, calcined at $823 \mathrm{~K}$ for 3 hours under dry air (10 
$\mathrm{ml} / \mathrm{min})$. The SBA-15 was synthesized using Pluronic P-123 ([OE]20-[OP]70-[OE]20) as structure-directing agent and TEOS like as silica source. Typically $3.01 \mathrm{~g}$ of P-123 was dissolved in $92 \mathrm{ml}$ of deionized water and $17.5 \mathrm{ml}$ of $\mathrm{HCl}$ (4 $\mathrm{M})$ at $323 \mathrm{~K}$ with stirring for $1 \mathrm{~h}$. Then, $7 \mathrm{ml}$ of TEOS was added dropwise without stirring for $24 \mathrm{~h}$. The mixture was maintained at $333 \mathrm{~K}$ for $48 \mathrm{~h}$ [6].

\section{Characterization}

To identify the crystalline compounds a Siemens D500 PW powder diffractometer coupled to a copper anode tube was used to obtain the X-ray diffraction pattern. The $\mathrm{Cu}-\mathrm{K}_{\alpha}$ radiation was selected with a diffracted beam monochromator. Radial distribution functions were calculated from X-ray diffraction patterns using a molybdenum anode X-ray tube coupled to a Siemens D500 powder diffractometer. The $\mathrm{K}_{\alpha}$ radiation was selected with a filter and data, measured by step scanning with a scintillation counter, were the input to the Radiale program [7].

A Kratky camera coupled to a copper anode X-ray tube was used to measure the Small Angle X-ray Scattering (SAXS) curves. Distance between sample and the linear proportional counter was $25 \mathrm{~cm}$; a $\mathrm{Ni}$ filter selected the copper $\mathrm{K}_{\alpha}$ radiation. The sample was introduced into a capillary tube. Intensity $\mathrm{I}(\mathrm{h})$ was measured for 9 minutes in order to obtain good quality statistics. The SAXS data were processed with the ITP program $[8,9]$ where the angular parameter $(h)$ is defined as $h=2 \pi \sin \theta / \lambda$, where $\theta$ and $\lambda$ are the X-ray scattering angle and the wavelength, respectively. The shape of the scattering particles was estimated from the Kratky plot, i. e., $\mathrm{h}^{2} \mathrm{I}(\mathrm{h})$ versus $\mathrm{h}$. The shape was determined depending on the Kratky curve shape, as, for instance, if the curve presents a peak the particles are globular [10]. If a shape can be assumed, the corresponding size distribution function may be calculated. Finally, it is often useful to estimate, from the slope of the curve $\log \mathrm{I}(\mathrm{h})$ versus $\log (\mathrm{h})$, the fractal dimension $[11,12]$. For this study, the background, obtained with the Porod plot, was subtracted from the experimental intensity. The $h$ interval was $0.07<\mathrm{h}<0.18 \AA^{-1}$. Note that by the Babinet principle, the SAXS may be due either to dense particles in a low-density

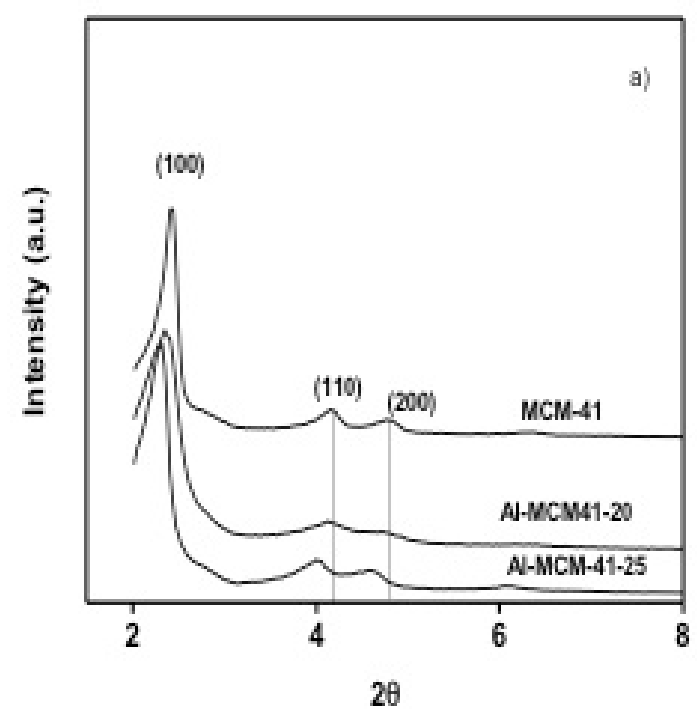

environment or to pores -or low-density inclusions- in a continuous high electron density medium. Therefore, in this work we will often use the word "heterogeneity" to term the scattering objects, which may be pores or particles.

Nuclear magnetic resonance (NMR) measurements were performed in a Bruker Avance 400 Spectrometer. The spectrometer was operated at a resonance frequency of 79.50 $\mathrm{MHz}$ for ${ }^{29} \mathrm{Si}$ MAS NMR spectroscopy. Spectra were recorded after single $\pi / 2$ excitation with repetition times of $6.5 \mathrm{~ms}$. Samples were spun at $5 \mathrm{kHz}$ and the chemical shifts were referenced to TMS. Spectra were obtained with 15000 scans.

Adsorption isotherms of $\mathrm{N}_{2}$ and Ar were obtained on an ASAP 2020 instrument after pretreating the samples under vacuum at $673 \mathrm{~K}$ overnight.

\section{RESULTS}

\section{Compounds}

The X-ray diffraction patterns of MCM-41, and AlMCM-41 are shown in Fig. (1a), where the angular interval is $0.5<2 \theta<11^{\circ}$. The peaks corresponding to each material appear in the expected angular position. The (100), (110) and (200) reflections indicate that these solids are well ordered with a hexagonal structure. The X-ray diffraction patterns of the MCM-41 and SBA-15 materials obtained in this work are similar to those presented in previous works $[13,14]$. They correspond to the well-known mesoporous silicates constituted by a large amount of non-crystalline material. The diffraction pattern of SBA-15 in Fig. (1b) shows the peak (100) slightly shifted towards lower $\theta$ angles. Hence, the interplanar distances and, therefore, the pore sizes must be larger.

\section{Structure}

The radial distribution function, (or pair correlation function) in a system of particles (atoms, molecules, colloids etc.), describes how the density varies as a function of the distance from a reference particle. It describes, then, the short-range order of the materials. The radial distribution function of MCM-41 sample is compared to those of silica

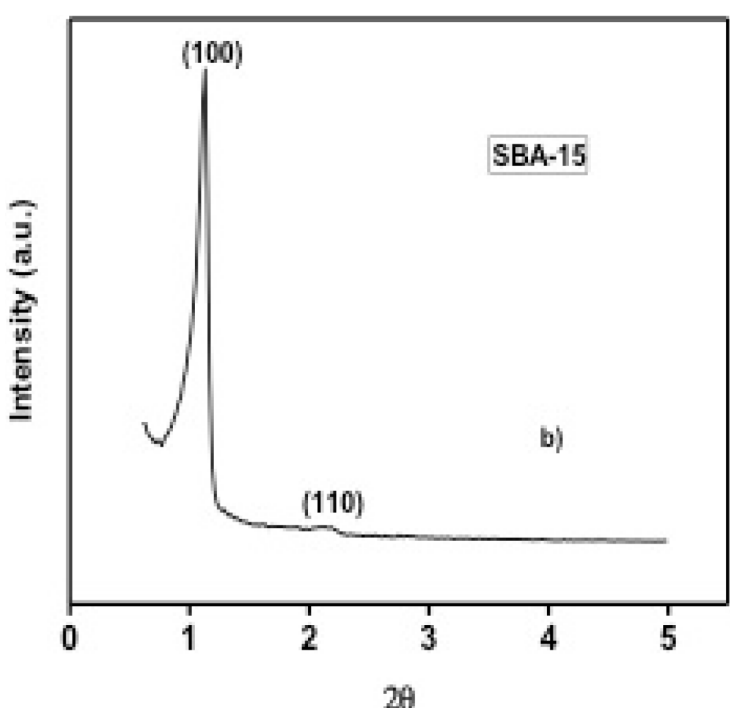

Fig. (1). a) X-ray diffraction pattern of MCM-41 and Al-MCM-41 samples and b) SBA-15. 
gel and quartz in Fig. (2). It is most interesting to compare these materials, as they are only constituted by silicon and oxygen but with different degrees of order. Silica gel main peaks, at $1.25,2.50$ and $3.65 \AA$, are attributed, as usual, to $\mathrm{Si}-\mathrm{O}, \mathrm{O}-\mathrm{O}$, and $\mathrm{Si}-\mathrm{Si}$ distances; they are different from those reported by Warren [15] 1.62, 2.65, and $3.2 \AA$. Indeed, the radial distribution function reported by Warren corresponds to a vitreous material whereas ours is a gel. It seems therefore that in amorphous silica, depending on the preparation method, the distance Si-O may vary as much as $0.37 \AA$. The same trend is followed by the O-O distance which in the gel is much shortershowing that, in this material, the building unit may be no more the regular tetrahedral unit found in glasses or common zeolites, but a distorted one.

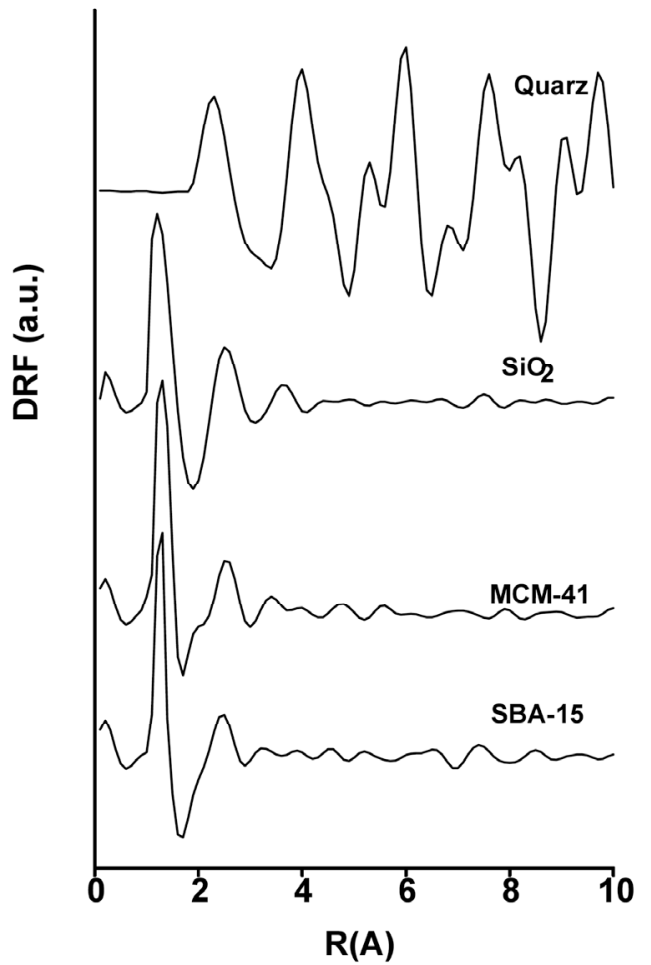

Fig. (2). Radial distribution functions of the, SBA-15, MCM-41, $\mathrm{SiO}_{2}$ and quartz.

The peaks appearing in SBA are located at radial distances of $1.20,1.90,2.50$ and $3.20 \AA$. The radial values 1.20 and $2.50 \AA$ reproduce the silica gel values. The differences between these two radial distribution functions are the $1.90 \AA$ shoulder, which was not observed in silica gel, and the peak at $3.20 \AA$, which is the $\mathrm{Si}-\mathrm{Si}$, shifted peak $3.60 \AA$ of silica. The angle $\mathrm{Si}-\mathrm{O}-\mathrm{Si}$ is, then, different in this material. The $1.90 \AA$ shoulder has to be attributed to an elongated distance $\mathrm{Si}-\mathrm{O}$ that is found together with the 1.20 Å distance.

In MCM41 radial distribution function, the first peak is located at $\mathrm{r}=1.20 \AA$ as in the SBA material, a well resolved shoulder appears at $r=1.90 \AA$, the peak corresponding to the $\mathrm{O}-\mathrm{O}$ distance is at $2.50 \AA$ and the one corresponding to the Si-Si distance at $3.40 \AA$. This silicate is, then, similar to the SBA material in the very short range but it differs in the $\mathrm{Si}-\mathrm{Si}$ distance, which determines the angle between tetrahedrons, and, therefore, the final structure. Note that in this sample, as in the SBA, the short and long Si-O distances are also found: 1.25 and $1.97 \AA$.

\section{Porosity}

The Kratky plot corresponding to silica gel obtained from SAXS data, Fig. (3), presents a broad peak typical of spherical or globular heterogeneities. Instead the shape of the MCM41 and SBA curves corresponds to cylindrically shaped heterogeneities. It is clear, then, that in the first case the heterogeneities are the globular particles of silica and that, in the other two, they correspond to pores in the porous material. Assuming such shapes, the size distribution was calculated, Fig. (4). Silica gel presents a broad particle size distribution with maxima located at radii of $15,50,80,120$ and $165 \AA$ Á. Instead, MCM41 has a monomodal pore size distribution whose maximum is at $80 \AA$. Pore sizes in SBA comprise an interval from $\mathrm{r}=10 \AA$ to $\mathrm{r}=80 \AA$, the maxima are found at 15,40 and $55 \AA$. Then, in SBA-15 a high percentage of pore radii are in the interval 5 to $40 \AA$, instead, in the MCM41, these sizes are not present.

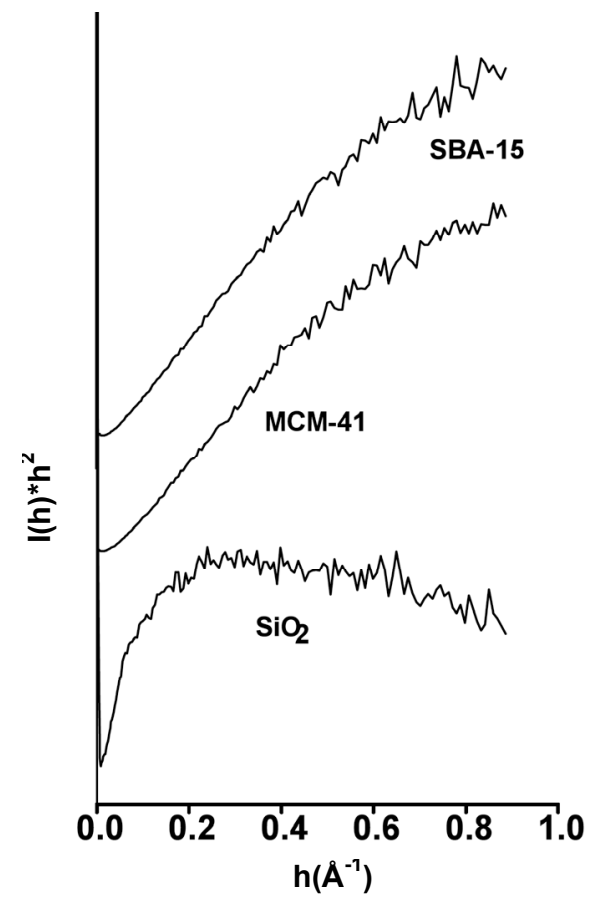

Fig. (3). Silica gel, MCM-41, and SBA-15 Kratky plots obtained from SAXS data.

\section{Fractal Dimension}

Fig. (5) compares the slope of the SAXS curves Log I (h) $v s$ Log $\mathrm{h}$. They are all parallel and correspond to a fractal dimension of 2.4. This value is between the fractal dimension $2.02 \pm 0.05$ for synthetic Faujasite and $2.9 \pm 0.40$ for silica 100 using as probe molecule n-alkanes as reported in $[11,12]$. It seems that zeolite pore walls are similar to the silica surface as far as SAXS fractal dimension is concerned.

In Fig. (6), the slopes (D) of the curves $\ln (\mathrm{V} / \mathrm{Vm})$ vs $\ln$ $(\ln (\mathrm{Po} / \mathrm{P})$ obtained, from the nitrogen gas adsorption experiments are compared in Table 1. Ds is the surface fractal dimension, it depends on the surface roughness. If the surface is smooth and flat, Ds turns out to be 2, a very disordered one has a Ds value of 3 , thus $2 \leq \mathrm{D} \leq 3[16,17]$. The obtained values are 2.80, 2.79 and 2.77 for the MCM41, AlMCM-41 20\% and Al-MCM-41 25\%, respectively. The 


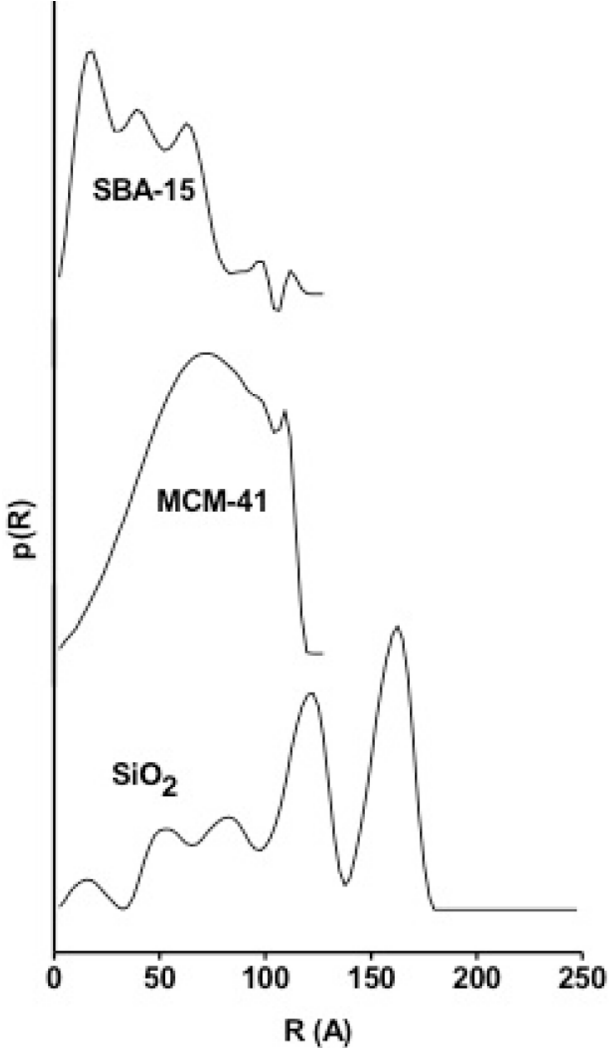

Fig. (4). Particle size distributions

surface is thus rough and irregular in the three samples although the MCM41 seems to be smoother. These values may be compared with those for silica xerogel and porous $\mathrm{AlOOH}$, reported by Brinker and Scherer [18], which were
$2.97 \pm 0.02$ and $2.57 \pm 0.04$, indicative of a mass fractal or extremely rough surface fractal. The value obtained for our silica gel, 1.84, does not agree with the values reported for other silica gels; Zhang et al. [19] have found a value of 2.77 if all the experimental data of mercury intrusion are considered but 2.10 and 2.38 depending on the porous scale. Furthermore, Vinogradova et al. [20] have shown the multifractal condition of TEOS derived silica whose fractal values may vary from 1.32 up to 2.4 .

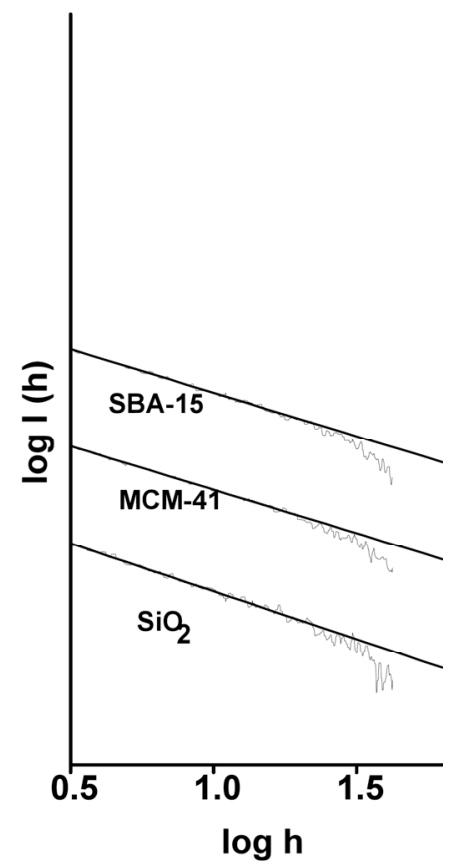

Fig. (5). $\log \mathrm{I}$ (h) vs $\log \mathrm{h}$ SAXS curves.

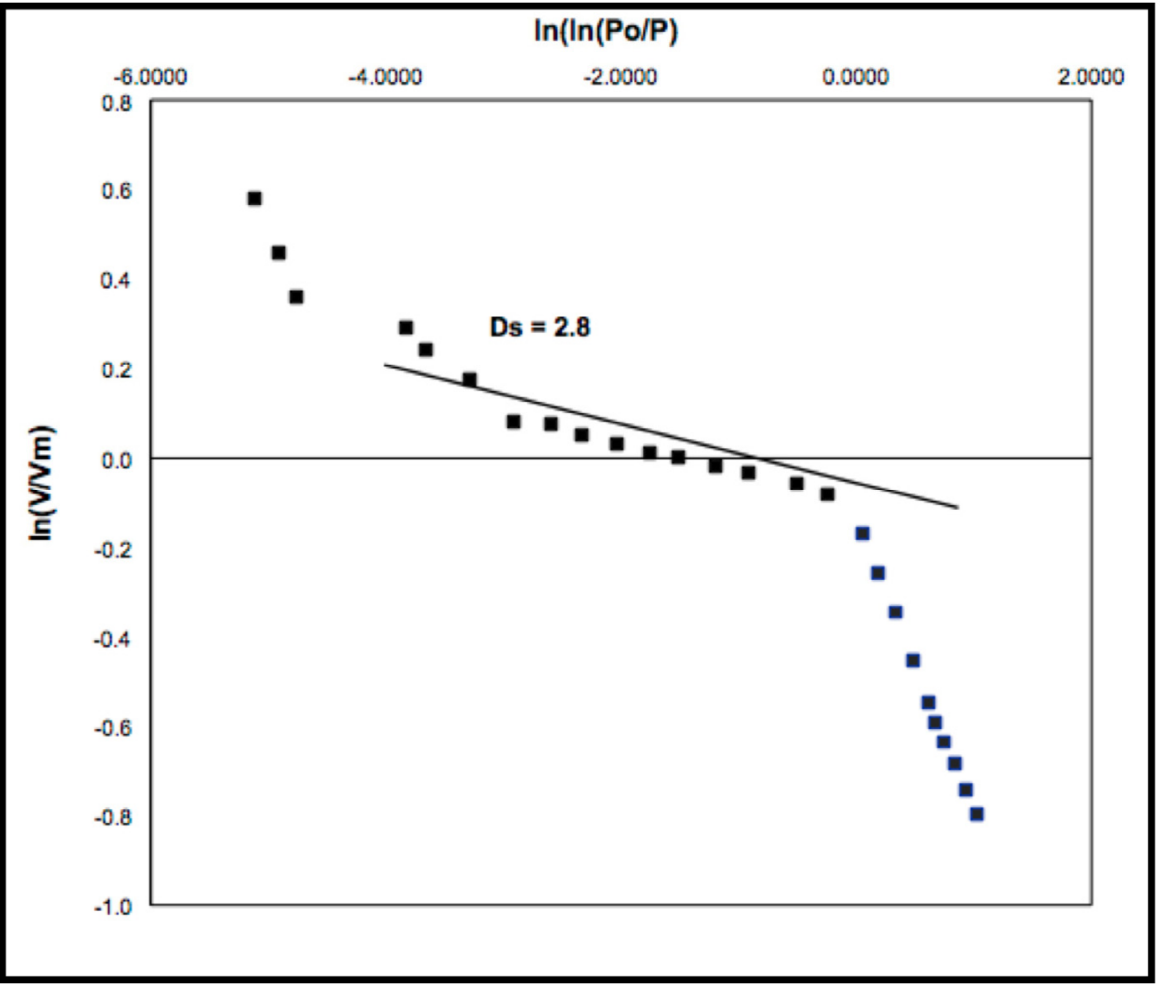

Fig. (6). MCM-41 slope (D) of the curve $\ln (\mathrm{V} / \mathrm{Vm})$ vs $\ln \left(\ln \left(\mathrm{p}_{0} / \mathrm{p}\right)\right)$ obtained from $\mathrm{N}_{2}$ gas adsorption. 
Hence, the following model emerges. Samples are homogeneous and similar as far as their SAXS fractal dimension is concerned, their volume connectivity is thus similar; instead, they may be differentiated through their surface fractal dimension determined by gas adsorption. The rougher surface is the Si-MCM-41. Silica surface fractal dimension is smaller than 2, then, the surface has to be constituted by chains. This proposition has been already discussed in previous works [19-21].

\section{Silicon Coordination}

To understand the coordination of the Si sites, the NMR results incorporate the study of a zeolite Faujasite $\mathrm{Y}$ for

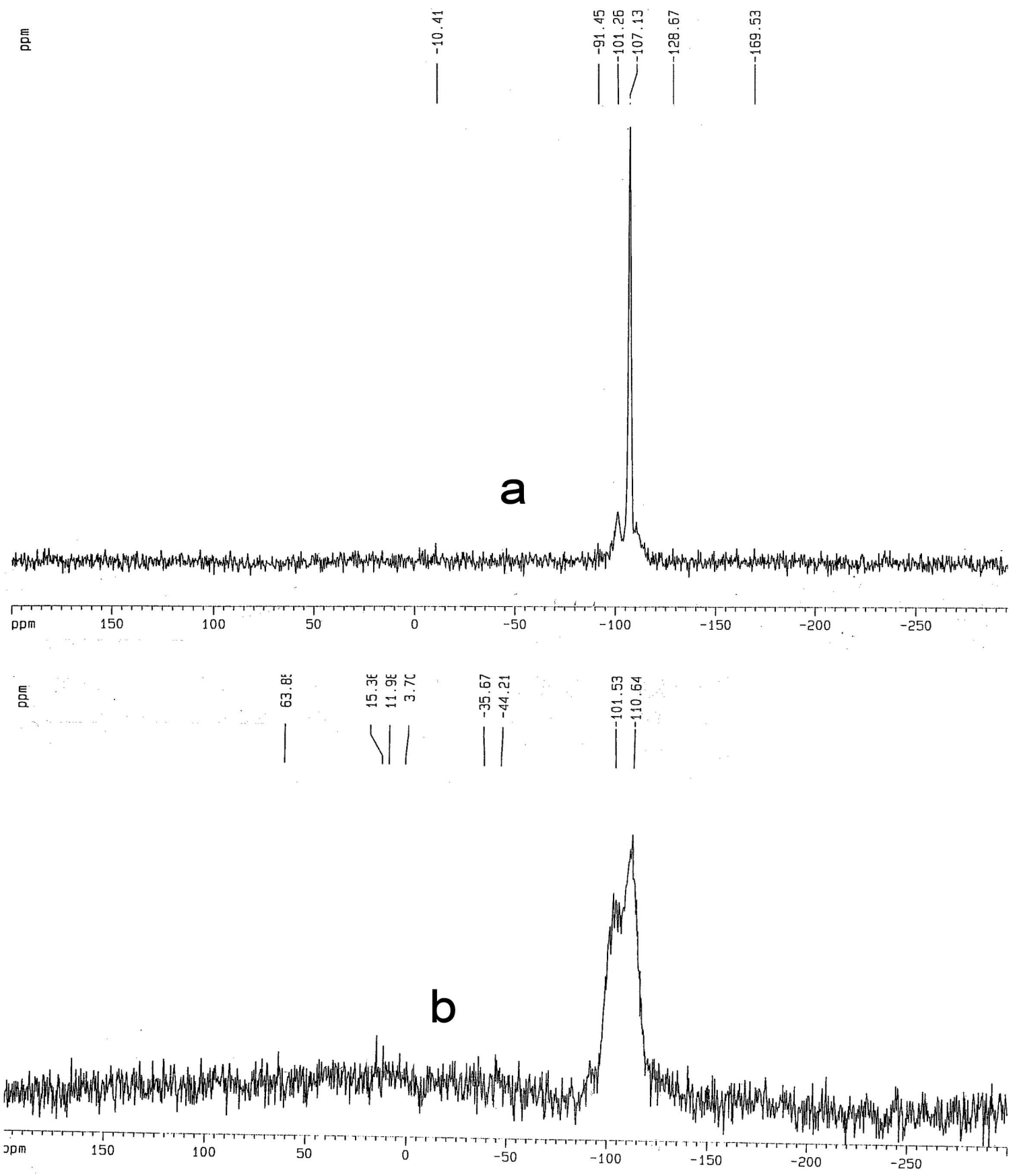

comparison purposes. Indeed, zeolites are built by $\mathrm{Si}(4 \mathrm{Al})$, $\mathrm{Si}(3 \mathrm{Al}), \mathrm{Si}(2 \mathrm{Al}), \mathrm{Si}(1 \mathrm{Al})$ and $\mathrm{Si}(0 \mathrm{Al})$ units, whose NMR signals are at $-86,-92,-97,-102$ and $-108 \mathrm{ppm}$, respectively [21]. $\mathrm{Si}(\mathrm{nAl})$ units build the zeolite framework, where $\mathrm{n}$ is the number of aluminums bonded to tetrahedral silicon. NMR spectra, Fig. (7a), show how, as expected, the shape of the Q4 peak is sharp and the intensity varies depending on the zeolite structure. Still, small shoulders and peaks are found at -101 ppm (Q3) and -106.23 ppm (Q4). In Fig. (7b), the NMR spectrum of MCM-41 sample is shown. If compared to zeolite $\mathrm{Y}$, a larger amount of Q3 $(2 \mathrm{Si} 3 \mathrm{O} 1 \mathrm{OH})$ is observed. The differences among those materials are shown by the shifts of Q3(2Si3O1OH) and Q4 (1Si4O). 
Table 1. $\quad \mathrm{N}_{2}$ Adsorption/Desorption Data and Surface Fractal (Ds) of the Samples

\begin{tabular}{|c|c|c|c|c|}
\hline & $\mathbf{S i O}_{\mathbf{2}}$ & $\mathbf{M C M - 4 1}$ & $\begin{array}{c}\text { Al-MCM-41- } \\
\mathbf{2 0}\end{array}$ & $\begin{array}{c}\text { Al-MCM-41- } \\
\mathbf{2 5}\end{array}$ \\
\hline \hline $\begin{array}{c}\text { Surface } \\
\text { Area BET } \\
\left(\mathrm{m}^{2} / \mathrm{g}\right)\end{array}$ & 192 & 1004 & 1136 & 1207 \\
\hline $\mathrm{D}_{\mathrm{s}}$ & 1.84 & 2.80 & 2.79 & 2.77 \\
\hline
\end{tabular}

\section{CONCLUSIONS}

Indirect information on the microscopic structure of mesoporous materials was obtained from experimental data. Samples are homogeneous and similar as far as their SAXS fractal dimension is concerned, their volume connectivity is thus similar; instead, they may be differentiated through their surface fractal dimension determined by gas adsorption. The rougher surface is the Si-MCM-41. Silica surface fractal dimension is smaller than 2, then, the surface has to be constituted by chains. In a study performed by means of fluorescence technique [3], it is proposed that the key step in the synthesis mechanism is the formation of silica prepolymers that are further polymerized and organized during the precipitation-aging sequence. It is likely that the prepolymer geometries are similar to those of the zeolite secondary building units, particularly those displaying 4- or 5 -member rings (the most frequent in zeolites). Further along the formation process, in the organization-polymerization step, the low probability of full 3D matching between the silanol groups explains the partially saturated or quite stressed structures. The NMR results confirm that mechanism as the observed amount of Q3 (2Si3O1OH) is higher than in zeolite $\mathrm{Y}$.

\section{CONFLICT OF INTEREST}

The authors confirm that this article content has no conflicts of interest.

\section{ACKNOWLEDGEMENT}

Declared none.

\section{REFERENCES}

[1] Kresge, M.C.; Leonowicz, W.T.; Roth, E.J.; Vartuli, J.C.; Beck, J.S. Ordered mesoporous molecular sieves synthesized by liquidcrystal template mechanism. Nature, 1992, 359, 710-712.

[2] Stein, A. Advances in microporous and mesoporous solidsHighlights of recent progress. Adv. Mater., 2003, 15, 763-775.

[3] Frasch, L.; Lebeau, B.; Soulard, M.; Patarin, L.; Zana, R. In situ investigations on cetyltrimethylammonium surfactant/silicate systems, precursors of organized mesoporous MCM-41-type siliceous materials. Langmuir, 2000, 16, 9049-9057.

[4] Taguchi, A.; Schüth, F. Ordered mesoporous materials in catalysis. Microporous Mesoporous Mater., 2005, 77, 1-45.

[5] Boveri, M.; Aguilar-Pliego, J.; Pérez-Pariente, J.; Sastre, E. Optimization of the preparation method of HSO-functionalized MCM-41 solid catalysts. Catal. Today, 2005, 107-108, 868-873.

[6] Trong On, D.; Desplantir-Giscard, D.; Donumah, C.; Kaliaguine, S. Perspectives in catalytic applications of mesostructured materials. Appl. Catal. A Gen., 2003, 253, 545.

[7] Aguillón, M.E.C.; Melo, B.J.A.; Silva, R.R.; Domínguez, J.M.; Aguilar, E.J. Structural properties of Carbides and Nitrides phases supported on SBA-15 and -16 from ammine salts precursors. Actas 20 NAM, 2007.

[8] Magini, M.; Cabrini, A. Programme en FORTRAN IV pour l'analyse des données expérimentales relatives à la diffusion des rayons $\mathrm{X}$ par des substances liquides, amorphes et microcristallisées. J. Appl. Cryst., 1972, 29, 702.

[9] Glatter, O. Comparison of two different methods for direct structure analysis from small-angle scattering data. J. Appl. Crystallogr., 1988, 21, 886 .

[10] Glatter, O.; Gruber, K. Small angle X-ray scattering hydrophilic and hydrophobic $\mathrm{SiO}_{2}$ particles. J. Appl. Crystallogr., 1993, 26, 512 .

[11] Kataoka, M.; Flanagan, J.M.; Tokunaga, F.; Engelman, D.M. Use of the X-ray solution scattering for protein folding study. In: Chanse, B.; Deisenhofer, J.; Ebashi, S.; Goodhead, D.T.; Huxley, H.E. Eds. Synchroton Radiation in the Biosciences. Clarendon Press: Oxford, UK, vol. 4, pp. 87-92, 1994.

[12] Harrison, A. Fractals in Chemistry. Oxford University Press Inc: New York, 1995.

[13] Medina-Valtierra, J.; Zaldivar, O.; Sánchez, M.A.; Montoya, J.A.; Navarrete, J.; de los Reyes, J.A. Kinetics of alkylation of benzene with isopropyl alcohol over Ce-exchanged NaX zeolite. Appl. Catal. A, 1998, 166, 387-392.

[14] Li, Q.; Brown, S.E.; Broadbelt, L.J.; Zheng, J.-G.; Wu, N.Q Photoisomerization of azobenzene from the trans- to the cis. Microporous Mesoporous Mater., 2003, 59, 105.

[15] Warren, B.E. X-ray Diffraction. Addison-Wesley: Reading MA; Boston, 1969.

[16] Martin, J.E.; Hurd, A. J. Scattering from fractals dimension are introduced, and from these basic concepts scattering functions are computed forsurface and mass fractals. J. Appl. Crystallogr., 1987, 20,61-78.

[17] Pernyeszi, T.; Dékány, I. Dramatic improvement in mechanical properties of GNs-reinforced HDPE nanocomposites. Colloid Polym. Sci., 2003, 281, 73 .

[18] Brinker, C.J.; Scherer, G. W. Sol-Gel Science. The Physics and Chemistry of Sol-Gel Processing. Academic Press: San Diego, USA, p. 532, 1990

[19] Zhang, B.; Lu, W.; Liu, X. Preparation of superhydrophobic surfaces on cotton textiles. Appl. Surf. Sci., 2006, 253, 1349.

[20] Vinogradova, E.; Moreno, A.; Lara, V.H.; Bosch, P. Multi-fractal imaging and structural investigation of silica hydrogels and aerogels. Silicon Chem., 2003, 2, 247.

[21] García, P.; Lima, E.; Aguilar, J.; Lara, V.H. Fractal extraframework species in De-aluminated LaY zeolites and their catalytic activity. Catal. Lett., 2009, 128, 385-391.

This is an open access article licensed under the terms of the Creative Commons Attribution Non-Commercial License (http://creativecommons.org/licenses/by-nc/ 3.0/) which permits unrestricted, non-commercial use, distribution and reproduction in any medium, provided the work is properly cited. 